\title{
Huaca Naranjal: Un centro de producción de CERÁMICA ESTILO YCHSMA EN EL VALle DE CHILlóN
}

Erik E. Maquera Sánchez*

\section{Resumen}

Recientes excavaciones en Huaca Naranjal han permitido registrar la recurrencia de grandes contextos de fragmentería cerámica los cuales han sido reconocidos como desechos de producción alfarera. El análisis de los contextos indica que la cerámica producida corresponde a tipos restringidos del estilo Ychsma resultado de un patrón de producción especializado que nos permite discutir la organización socioeconómica de la población. La identificación de un centro de producción de cerámica estilo Ychsma en el valle bajo del Chillón nos plantea el problema sobre su identificación política, considerando su proximidad a los etnohistóricamente definidos Colli.

\section{Palabras Claves}

Contextos de fragmentería cerámica, producción, economía, Períodos Intermedio Tardío y Horizonte Tardío.

\begin{abstract}
Recent excavations at Huaca Naranjal have registered the recurrence of large pottery sherd contexts, which have been recognized as a waste of pottery production. The analysis of the contexts indicates that the ceramics produced is part of restricted types of Ychsma style result of a pattern of specialize production, that enables the discussion of the socio-economic organization of the population. The recognition of an Ychsma style production pottery center in the lower valley of Chillon poses the problem on its political identification, considering its proximity to the ethnohistoric defined Colli.
\end{abstract}

\section{Keywords}

Pottery sherd contexts, production, economy, Late Intermediate and Late Horizon periods

* Universidad Nacional Mayor de San Marcos. Correo electrónico: erikleif18@yahoo.com 


\section{INTRODUCCIÓN}

Durante los meses de marzo y abril del año 2006 se realizó el Proyecto de Evaluación Arqueológica Naranjal, dirigido por el Dr. Miguel Cornejo Guerrero. Paralelamente a la ejecución de los trabajos, el Dr. Cornejo me alentó a realizar un análisis del material cerámico con la finalidad de indagar sobre la posible presencia de un taller de alfareros en el sitio. En esta oportunidad presentamos las excavaciones y el análisis de los materiales de Huaca Naranjal, un centro de producción de cerámica de finales del Periodo Intermedio Tardío y Horizonte Tardío, ubicado en el valle bajo del río Chillón.

\section{UBICACIÓN}

Huaca Naranjal se encuentra ubicada en la margen izquierda del valle bajo del río Chillón, políticamente se localiza en el Distrito de Los Olivos, dentro del Asentamiento Humano Armando Villanueva del Campo (Fig.1).

El área alrededor del montículo, hasta la década del 70, fue parte de los terrenos de cultivo de la Hacienda Naranjal, en la actualidad las zonas agrícolas han desaparecido, tomando su lugar densas urbanizaciones y avenidas que han reducido sus dimensiones, ejemplo de ello ha sido el corte lateral efectuado por el municipio de Los Olivos para la ampliación de la Avenida Huandoy. Según la fotografía aérea del sitio, existieron varios montículos pequeños alrededor de Huaca Naranjal, el último de ellos ubicado a 200 metros al oeste fue nivelado a comienzos de los años 90 para la construcción de una urbanización. Es muy probable que el asentamiento haya sido un gran complejo con una extensión aproximada de 40 hectáreas. Evidencia de material arqueológico pueden encontrarse hasta 400 metros alrededor del montículo principal, tal como se manifiesta cada vez que se excavan zanjas para la construcción de viviendas, excediendo así la zona arqueológica intangible propuesta por el INC en 1986.
El montículo principal tiene actualmente una longitud de 130 metros con una altura de 10 metros y se encuentra orientado exactamente hacia el Noreste. La arquitectura del montículo se encuentra cubierta de tierra, aunque en la cima y en el corte producido por la Avenida Huandoy es posible observar algunos muros de tapia. Recurriendo de nuevo a la fotografía aérea y a la versión de muchos vecinos, sabemos que el montículo se prolongaba 50 metros hacia el noreste a modo de una gran plataforma. Aproximadamente hace diez años, esta parte del montículo fue destruida con maquinaria pesada con la finalidad de nivelar el área y de vender la tierra a una ladrillera local, finalmente el socavón ocasionado por la extracción de tierra alcanzó un metro por debajo de la superficie actual y fue rellenado con desmonte de tierra y basura. Es en esta zona en donde el Proyecto Arqueológico Naranjal realizó excavaciones de prueba con la finalidad de evaluar el potencial arqueológico del área afectada.

\section{ANTECEDENTES Y PROBLEMÁTICA}

Sobre los antecedentes del sitio es poco lo que se puede decir, ya que Huaca Naranjal no ha sido objeto de ninguna excavación o investigación arqueológica previa, ni aparece en los catastros arqueológicos publicados por Horkheimer (1965), Bonavía (1966), Milla Villena (1974), Rogger Ravines (1985), y FAUA-UNIFORD (1989). El sitio sólo cuenta con planos de delimitación realizados por el INC.

De esta forma nuestras excavaciones en el año 2004 y 2006, dirigidas por Miguel Cornejo Guerrero, constituyen las primeras investigaciones que tuvieron como objetivo precisar la filiación social y cronológica, así como el rol del asentamiento en el esquema general del valle.

Un primer acercamiento a estas problemáticas han sido los resultados del Proyecto de Evaluación Arqueológica con Excavaciones Huaca Naranjal (Cornejo 2004), en donde se 


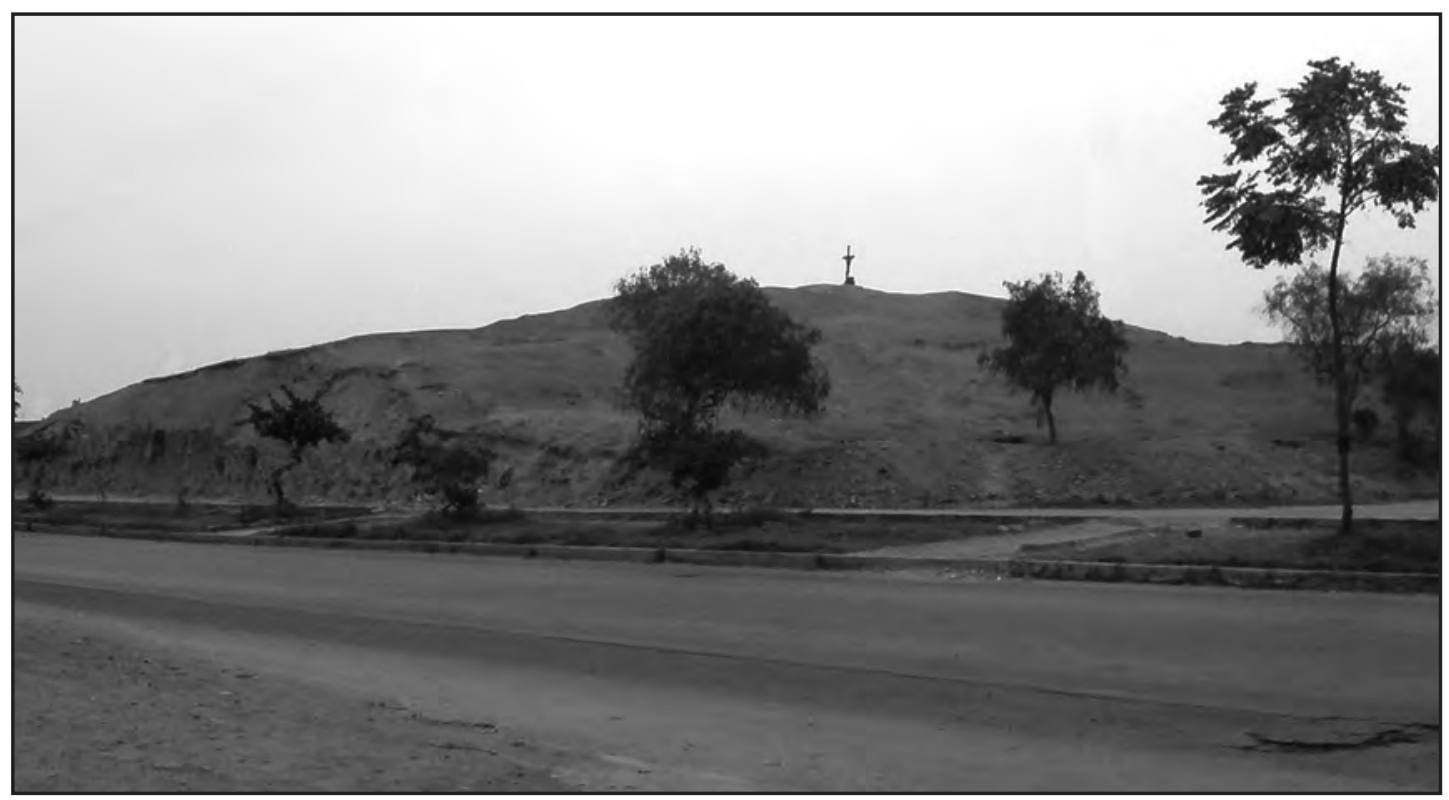

Figura1. Vista desde el sur de Huaca Naranjal.

realizaron 25 pozos de cateo distribuidos aleatoriamente en todo el asentamiento, cuyos materiales permitieron sostener que la ocupación de Huaca Naranjal correspondía a un taller de alfareros del Período Intermedio Tardío y tuvo finalmente una ocupación Inca, resaltando el hallazgo de un pequeño aríbalo de factura local (Cornejo 2004).

A partir de la hipótesis de Cornejo (2004) sobre la presencia de un taller de alfareros en Huaca Naranjal, se realizaron las excavaciones del año 2006 en la cual me dispuse a realizar el análisis de los materiales excavados con el fin de identificar un centro de producción de cerámica y reconocer la filiación social del mismo.

El tema de la producción de cerámica en los Andes ha tenido importantes avances en la década pasada (Shimada 1994), sin embargo estos avances no han abarcado el área de la costa central para ninguno de los períodos, ya que ningún taller o centro de producción de cerámica ha sido publicado, aunque muy probablemente hayan sido excavados. Según Rice (1987) uno de los problemas al abordar el estudio de la pro- ducción de cerámica se encuentra en la escasez de los talleres en el registro arqueológico, así como la ausencia de excavaciones en los mismos. Otro problema es que la investigación de la producción de cerámica metodológicamente esta condicionada a categorías como la escala de la producción, el grado de especialización, entre otras, cuyas variables en la mayoría de los casos son invisibles en el registro arqueológico o requieren necesariamente de excavaciones extensivas. Así, los estudios sobre la producción de cerámica han sido abordados indirectamente a través del estudio de la manufactura de las piezas cerámicas y no desde los contextos de producción. Sólo en años recientes se han desarrollado investigaciones que intentan identificar las áreas de actividad productiva de modo que se puede entender directamente la organización de estas (Shimada 1994).

En esta investigación consideramos que la producción de cerámica se define por las relaciones socioeconómicas que involucran la práctica de la manufactura, así, la producción como actividad económica se encuentra adscrita a un 
contexto social y político, en donde la manufactura se vincula directamente a patrones de distribución y consumo (Rice 1987: 168).

La identificación de un centro de producción de cerámica en el registro arqueológico es algunas veces difícil. Para ciertas sociedades, la producción pudo haberse desarrollado en sitios pequeños como aldeas, las cuales no han sobrevivido hasta nuestros días por procesos de expansión agrícola y urbana. Según Carmichael (1994), en algunas sociedades la manufactura de cerámica se realizaba a nivel doméstico, incluso en sociedades estatales como Nazca. Dean Arnold (1994), a partir de sus estudios etnográficos, también piensa que la unidad productiva de la manufactura de cerámica en los Andes es la unidad doméstica.

Otro obstáculo en la identificación de un centro de producción lo constituye la conservación de los materiales, algunas herramientas pueden haber estado confeccionadas de materiales perecibles como madera, caña, hueso, fibras. Si se añade a este problema, la excesiva acidez de algunos suelos entonces es seguro que ninguno de estos materiales podría conservarse, este es el caso de los suelos de Naranjal, en donde los objetos de madera y otros materiales perecibles sólo han dejado improntas. De otro lado, algunas instalaciones como hornos no necesitan de una estructura elaborada, se ha demostrado por ejemplo en los Andes, que la mayoría de hornos están constituidos por simples concavidades en la tierra, las cuales en excavaciones restringidas pueden no ser advertidas.

A pesar de las dificultades en la identificación de un centro de producción de cerámica, algunos materiales y contextos pueden dar los primeros indicios de tal presencia. Estos materiales y contextos pueden agruparse bajo las siguientes categorías: instalaciones, materia prima, herramientas de manufactura, desechos de producción y otros materiales como desechos domésticos (Cuadro 1).

Las categorías del Cuadro 1, incluyen una serie de indicadores que usualmente se encuentran asociados a los talleres de producción de

Cuadro 1. Indicadores de un área de producción de cerámica

\begin{tabular}{|l|l|l|l|l|}
\hline Instalaciones & Materia Prima & $\begin{array}{l}\text { Herramientas } \\
\text { de } \\
\text { manufactura }\end{array}$ & $\begin{array}{l}\text { Desechos de } \\
\text { producción }\end{array}$ & $\begin{array}{l}\text { Otros } \\
\text { materiales }\end{array}$ \\
\hline Hornos & Arcillas & Paletas & $\begin{array}{l}\text { Gran cantidad } \\
\text { de fragmentos } \\
\text { de cerámica }\end{array}$ & Vasijas usadas \\
\hline $\begin{array}{l}\text { áreas de mezcla } \\
\text { de arcilla }\end{array}$ & temperantes & moldes & $\begin{array}{l}\text { Fragmentos } \\
\text { recocinados }\end{array}$ & $\begin{array}{l}\text { Restos de } \\
\text { alimentos }\end{array}$ \\
\hline $\begin{array}{l}\text { áreas de secado } \\
\text { de de }\end{array}$ & combuatible & $\begin{array}{l}\text { alisadores } \\
\text { desbastadores } \\
\text { crudos }\end{array}$ & $\begin{array}{l}\text { Utensilios } \\
\text { (batanes, manos } \\
\text { de moler) }\end{array}$ \\
\hline $\begin{array}{l}\text { áreas de } \\
\text { almacenamiento } \\
\text { de combustible }\end{array}$ & pigmentos & pinceles, etc. & & \\
\hline $\begin{array}{l}\text { área } \\
\text { manufactura }\end{array}$ & & & \\
\hline
\end{tabular}


cerámica, siendo los materiales más hallados las herramientas de manufactura y los desechos de la producción, en particular para nuestro caso. Así mismo, debido a que nuestras excavaciones en Huaca Naranjal fueron restringidas sólo detectamos indicios de posibles instalaciones, si duda éstas requieren de excavaciones más extensivas.

\section{ESPACIO GEOGRÁFICO}

La materia prima para la manufactura de cerámica es uno de los factores relacionados al contexto del espacio geográfico. Según Arnold (1994: 484) a partir de una muestra tomada de alfareros de todo el mundo, la distancia que recorre un alfarero para la obtención de materia prima es en promedio de $1 \mathrm{~km}$., no excediendo los $7 \mathrm{~km}$ en busca de otros recursos como agua, combustibles, arcillas y temperantes.

Huaca Naranjal se encuentra en el valle bajo del río Chillón, en la región denominada por Tosi (1960) Llanura Desértica Subtropical, cuyo clima árido y ausente de lluvias es ideal para la manufactura de cerámica.
En Huaca Naranjal el estrato estéril consistió de un profundo banco de arcilla directamente sobre el cual yace la primera ocupación del sitio a finales del período Intermedio Tardío. Así mismo, es interesante observar que no existe ningún estrato u horizonte de tierra cultivable, por lo que se infiere que el área de Naranjal no formó parte del espacio productivo agrícola del valle para ninguna de las sociedades precedentes. De tal manera, es muy posiblemente que este banco de arcilla motivara el emplazamiento del centro de producción en esta área.

Por otro lado, la zona de Naranjal tiene la particularidad de tener una napa freática superficial que afloraba en el denominado puquio de Naranjal, el cual ha sido registrado en mapas y documentos coloniales y republicanos (Cerdan y Pontero 1793, Turner y Bravo 1907). Este puquio se encuentra en las inmediaciones del asentamiento, de forma similar que en el asentamiento de Aznapuqio. Lamentablemente el puquio desapareció paulatinamente hasta los 70'. Según el esquema de Arnold, en Huaca Naranjal las condiciones de producción se cum-

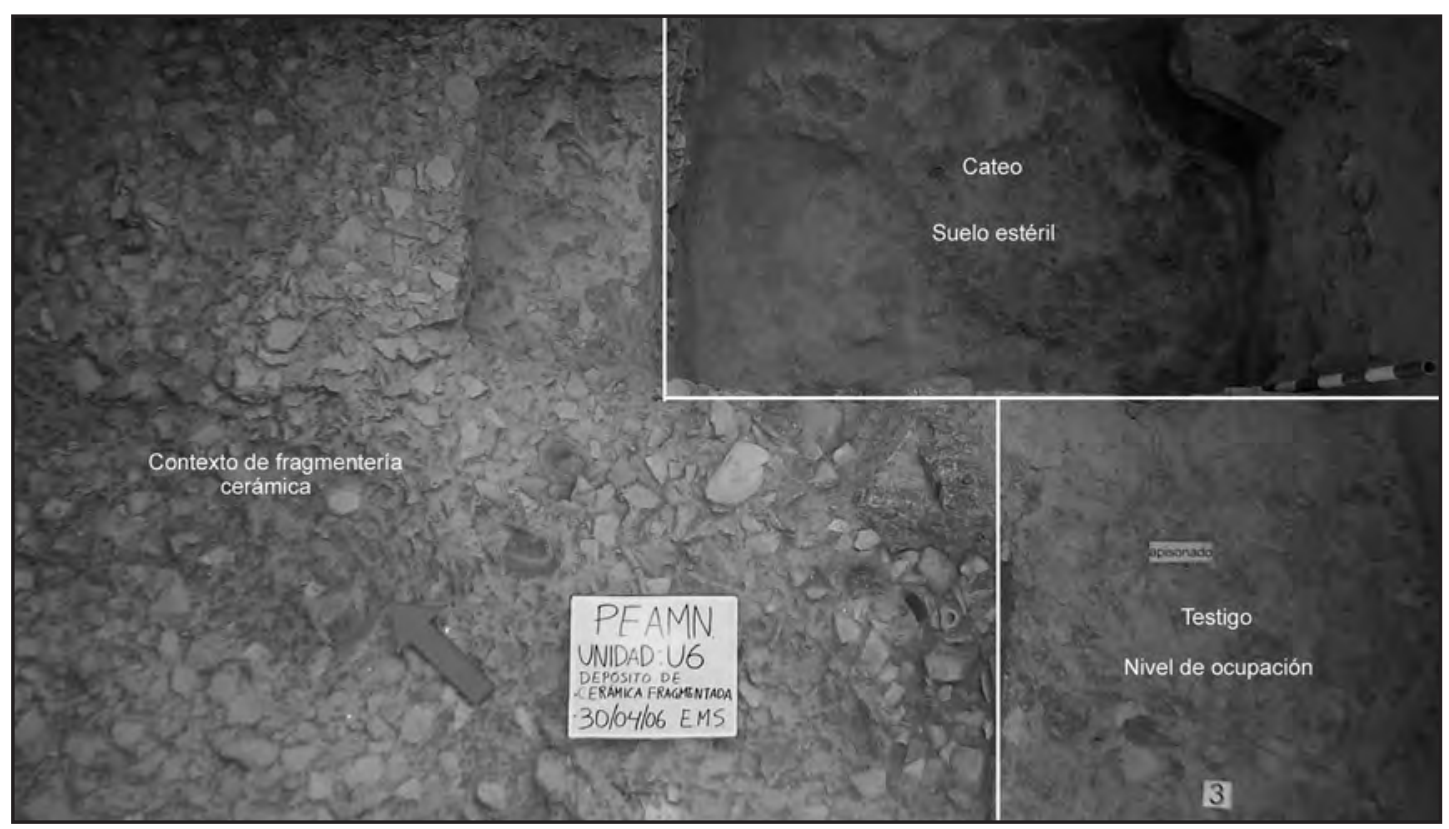

Figura 2. Depósito de cerámica fragmentada. 


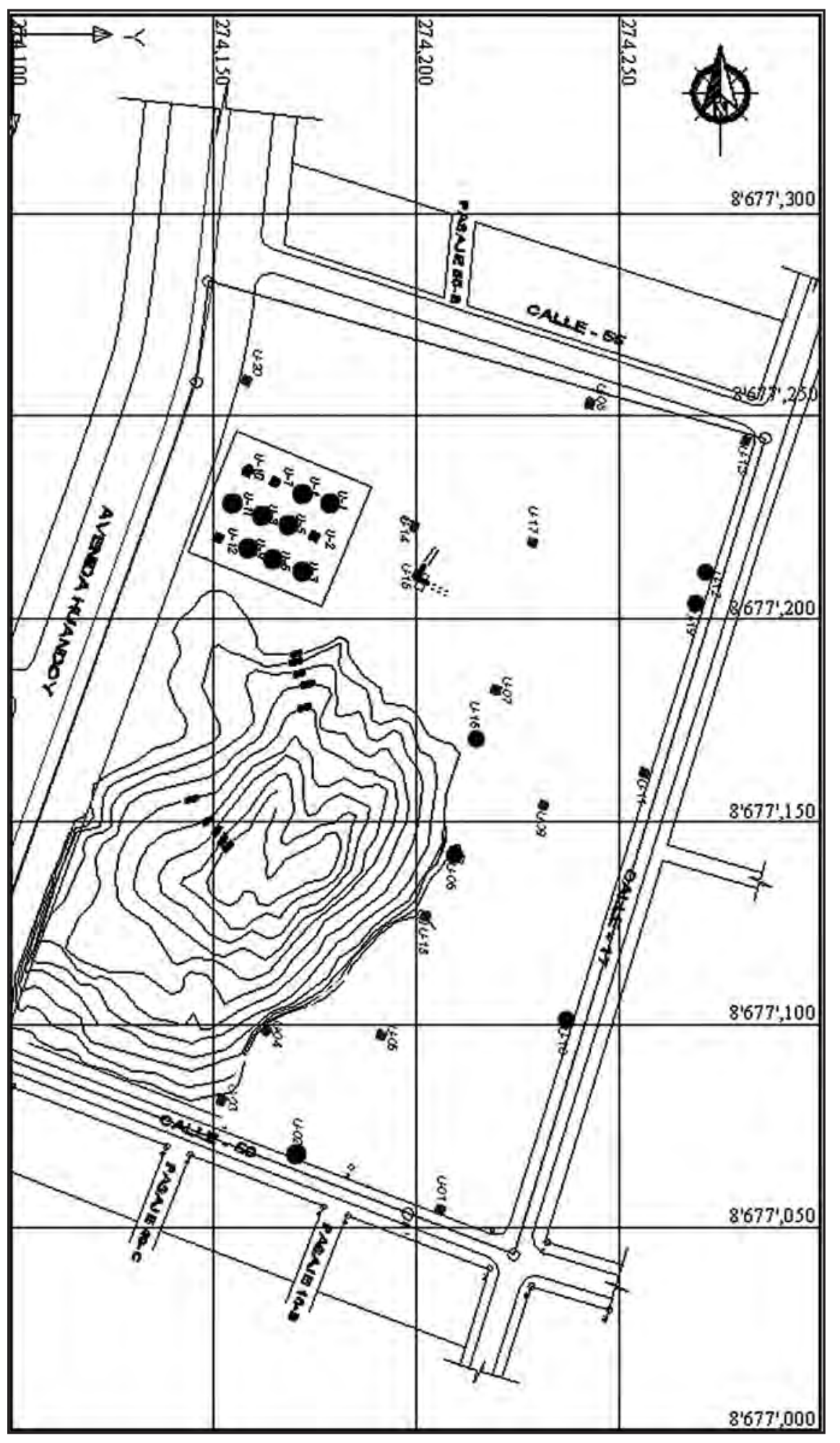

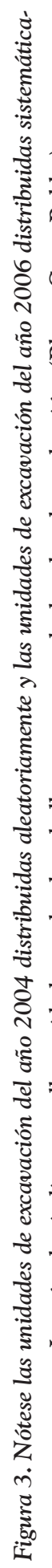


plen al disponer de las materias primas básicas para la manufactura tales como un gran banco de arcilla y agua del puquio aledaño. El combustible probablemente fue extraído de las zonas agrícolas y de los arbustos que habrían crecido aprovechando las napas freáticas. Otras arcillas y temperantes se encontrarían alrededor de una radio de $7 \mathrm{~km}$.

\section{LAS EXCAVACIONES}

Durante los meses de marzo y abril del año 2006 se realizó la excavación de 12 pozos de cateo de $2 \mathrm{~m}$. x $2 \mathrm{~m}$. distribuidos sistemáticamente cada 8 metros (Fig.3). Estos se ubicaron en la parte norte de la Huaca Naranjal y debajo de lo que alguna vez fue su plataforma norte, destruida por trabajos de nivelación y extracción de tierra con maquinaria pesada, que disturbaron parcialmente los depósitos arqueológicos subyacentes hasta una profundidad de $1 \mathrm{~m}$. por debajo de la superficie actual del área (Fig.4).

El trabajo de campo nos ha permitido identificar la recurrencia de depósitos de cerámica fragmentada (Fig.2), los cuales estuvieron presentes en ocho de las doce unidades de excavación: $1,3,4,5,6,8,9$ y 11 . Se trata en su gran mayoría de fragmentos no diagnósticos, seguidos de bordes de ollas y cántaros predominantemente sin decoración, así como un menor número de fragmentos de figurinas y moldes. Sólo dos especimenes fueron hallados completos: una figurina y un pequeño molde en la Unidad 6 (Figs. 7 y 9). Así mismo, estos contextos presentan abundantes fragmentos recocinados, alisadores, terrones quemados, temperantes, material lítico (herramientas), entre otros. A juzgar por tales indicadores, estos depósitos de cerámica fragmentada corresponden a desechos de producción.

Si bien no se pudieron definir áreas de actividad debido a la metodología de excavación (pozos de cateo de $2 \times 2$ m.), sí hemos detectado niveles de ocupación que presentan superficies rojizas, ennegrecidas y blanquecinas, además de densas acumulaciones de ceniza (posibles hornos), y mesas de trabajo de piedra. Adicio-

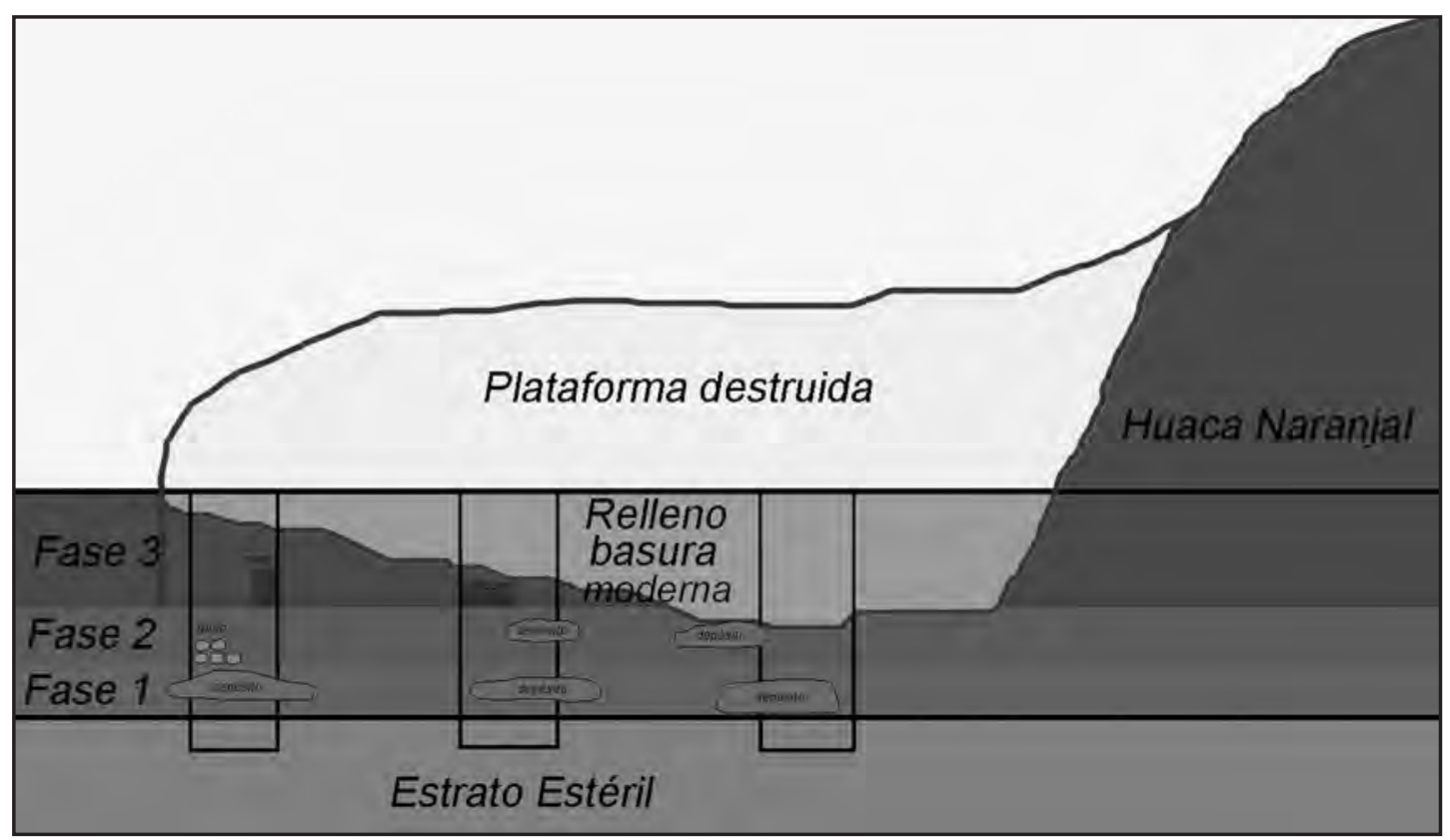

Figura 4. Estratigrafía de Huaca Naranjal, ubicación de los desechos de producción y elementos arquitectónicos. 
Cuadro 1. Tipos de vasijas definidas.

\begin{tabular}{|l|l|c|}
\hline \multicolumn{1}{|c|}{ Tipo } & \multicolumn{1}{|c|}{ Descripción } & Porcentaje \\
\hline Tipo 1 & Cántaro de cuello recto divergente & $3.4 \%$ \\
\hline Tipo 2 & Cántaro de cuello cóncavo divergente & $7.8 \%$ \\
\hline Tipo 3 & Olla de cuello recto & $11.5 \%$ \\
\hline Tipo 4 & Olla sin cuello y labio en "Forma de T" & $46 \%$ \\
\hline Tipo 5 y 6 & Olla de cuello recto y labio "lenticular" & $2.8 \%$ \\
\hline Tipo 7 & Olla de cuello recto y labio "media flecha" & $0.7 \%$ \\
\hline Tipo 8 & Cántaro de cuello convergente & $4.7 \%$ \\
\hline Tipo 9 & Olla de cuello "abombado" & $4.5 \%$ \\
\hline Tipo 10 & Olla de cuello compuesto & $3.3 \%$ \\
\hline Tipo 11 & Olla de cuello convexo & $1.7 \%$ \\
\hline Tipo 12 & Cántaro de cuello convexo divergente & $3.7 \%$ \\
\hline Tipo 13 & Olla de cuello recto divergente & $9.4 \%$ \\
\hline Casos únicos & Miniaturas, cuencos, "canchero", entre otros. & $0.5 \%$ \\
\hline
\end{tabular}

nalmente algunos niveles de ocupación contuvieron una regular cantidad de desechos de consumo.

\section{ESTRATIGRAFÍA}

A partir de los 12 pozos de cateo se han detectado tres grandes conjuntos de estratos que hemos denominado Fases 1, 2 y 3 (Fig.4). La primera fase está asociada a 6 contextos de desechos de producción, la segunda fase está asociada a 2 contextos de desechos de producción y a arquitectura de piedras canteadas, y la tercera asociada a la construcción de arquitectura de tapia y contextos de relleno, los cuales fueron hallados en siete pozos de cateo. Los depósitos de cerámica sólo aparecen en las dos primeras fases del asentamiento asociadas al estilo Ychsma Tardío A, mientras que la Fase 3 esta asociada al inicio de la construcción de arquitectura monumental asociada a cerámica de la fase Ychsma Tardío B según la secuencia de Vallejo (2004).

\section{La muestra}

Se analizó una muestra cerámica de 3719 fragmentos diagnósticos, de un total de 22204 fragmentos excavados en 12 pozos de cateo, es decir un 16\% del total, con la finalidad de hallar el repertorio de tipos de vasijas y tipos de pasta, así como sus relaciones.

La mayoría de estos eran reconstruibles, pero la escasez de tiempo y recursos no permitió la restauración de las vasijas, ni la ejecución de otros análisis como el de decoración y acabado de superficie, necesarios para la definición de alfares.

\section{Resultados del análisis}

Se definieron 13 tipos de vasijas estadísticamente frecuentes que pueden ser asociadas al estilo Ychsma Tardío B según la secuencia de Vallejo (2004), en donde el Tipo 4 fue el que obtuvo la mayor frecuencia (Cuadro 3, Fig.5). Los tipos identificados que presentamos a continuación corresponden sólo a los depósitos de cerámica fragmentada de las Fases 1 y 2, es decir de la Fase Ychsma Tardío A (Cuadro 2 y 3).

Adicionalmente a estos tipos de vasijas, los depósitos de cerámica fragmentada contienen también, figurinas antropomorfas y zoomorfas, generalmente fragmentadas, aunque algunas de ellas fueron depositadas enteras sobre los contextos a manera de ofrenda (comunicación personal Cornejo); gran cantidad de fragmentos 
Cuadro 3. Frecuencia de tipos de vasijas, nótese que el Tipo 4 es el de mayor producción.
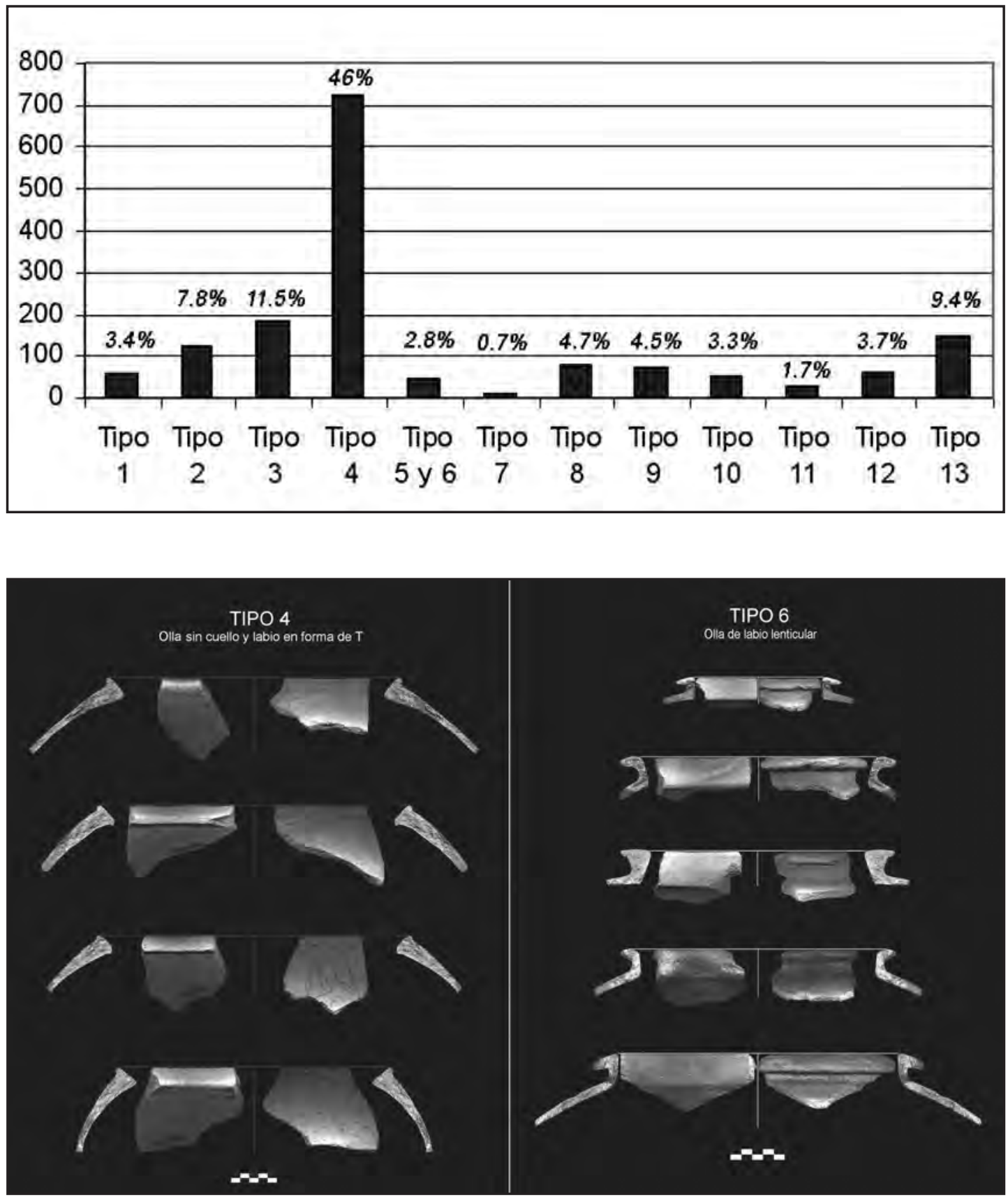

Figura 5. Tipos de vasijas manufacturadas en Huaca Naranjal. 


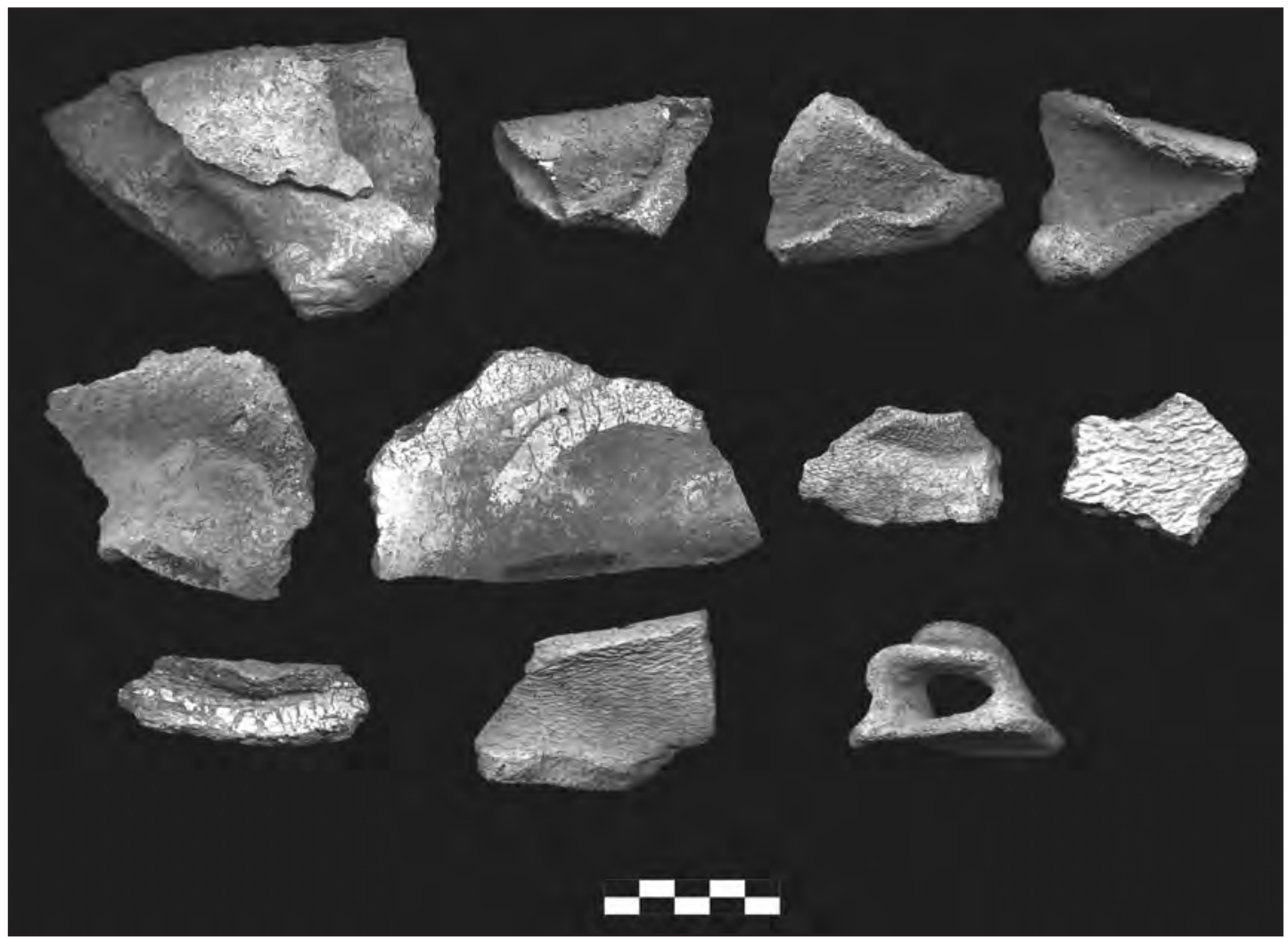

Figura 6. Fragmentos recocinados.

recocinados, llegando a alcanzar 900 fragmentos en un solo contexto (Fig 6); moldes, gran parte de ellos fragmentados (Figs. 8 y 9); alisadores tanto de cerámica como de material lítico; terrones de arcilla quemada; lajas grandes y medianas, probablemente bases o herramientas, que pueden alcanzar más de 600 en un solo contexto; gran cantidad de cantos rodados fragmentados, muchos de ellos con huellas de uso como manos de moler, pueden alcanzar el número de 500 en un solo contexto; formaciones de carbonato de calcio, que se encuentran originalmente en los bancos de arcilla de Naranjal, los cuales fueron utilizados como temperantes; y concentraciones de moluscos. Similares depósitos de cerámica fragmentada han sido reportados en Aznapuquio (Ludeña 1975, Espinoza este volumen), siendo también interpretados como desechos de producción de cerámica. Los depósitos analizados contienen además una cantidad importante de vasijas con evidencias de uso. La recurrencia de estos desechos en la Fase 1 y su continuidad en la Fase 2

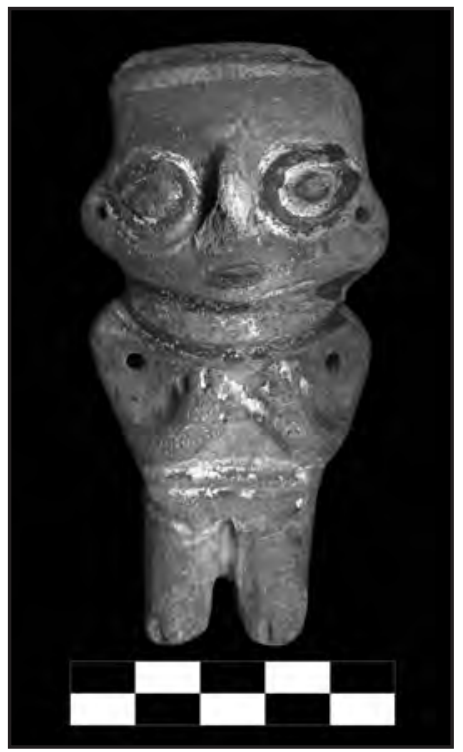

Figura 7. Figurina. 
nos permite considerar que estos contextos corresponden tanto al cierre de ciclos de producción como de uso de vasijas domésticas.

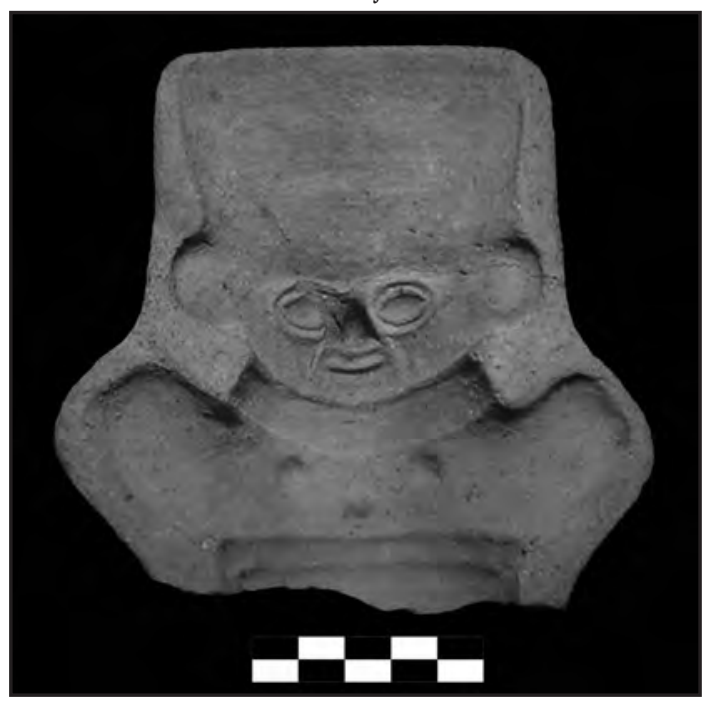

Figura 8. Fragmento de molde de figurina.
A partir del análisis morfológico se identificaron básicamente ollas y cántaros, y de forma marginal algunos fragmentos de miniaturas y un canchero. Los datos indican que en Naranjal se producían de forma exclusiva vasijas domésticas y de almacenaje del estilo Ychsma, es decir ollas y cántaros (Cuadro 4), quedando totalmente ausente la manufactura de vasijas de servicio o de cerámica fina. Esto puede interpretarse como el patrón de una producción especializada orientada a un determinado grupo de consumidores.

En efecto, los tipos cerámicos "finos" tales como los cántaros gollete y las vasijas de servicio fueron manufacturados en otros talleres especializados y orientados a satisfacer probablemente la demanda de las elites. iSignifican estas evidencias que la manufactura de ciertos tipos cerámicos se encontraba restringida por alguna autoridad?, o ise trataba de grupos especializados con cierta autonomía?

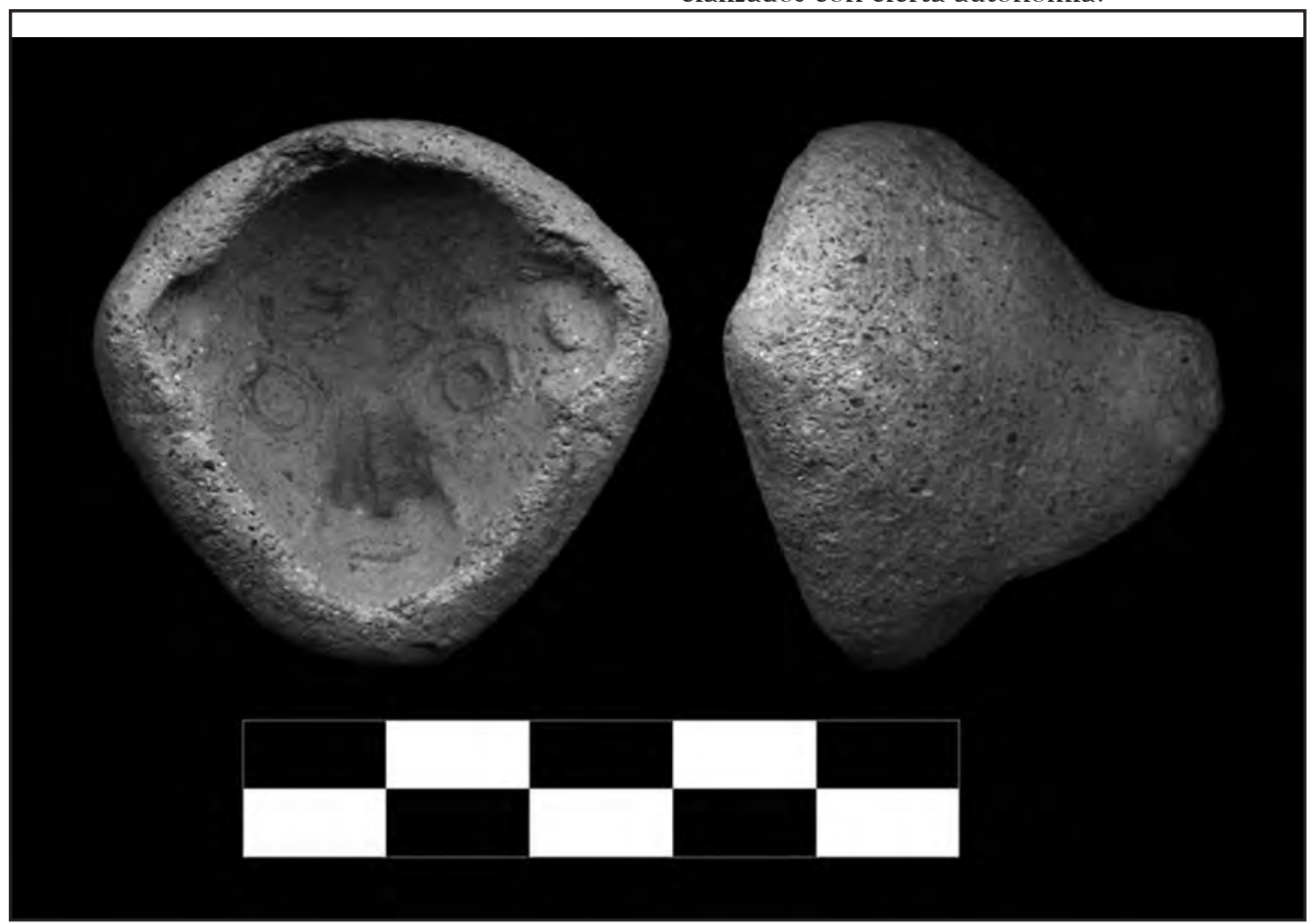

Figura 9. Pequeño molde para aplicaciones. 


\section{Cuadro 4.}

Frecuencia de categorías morfofuncionales.
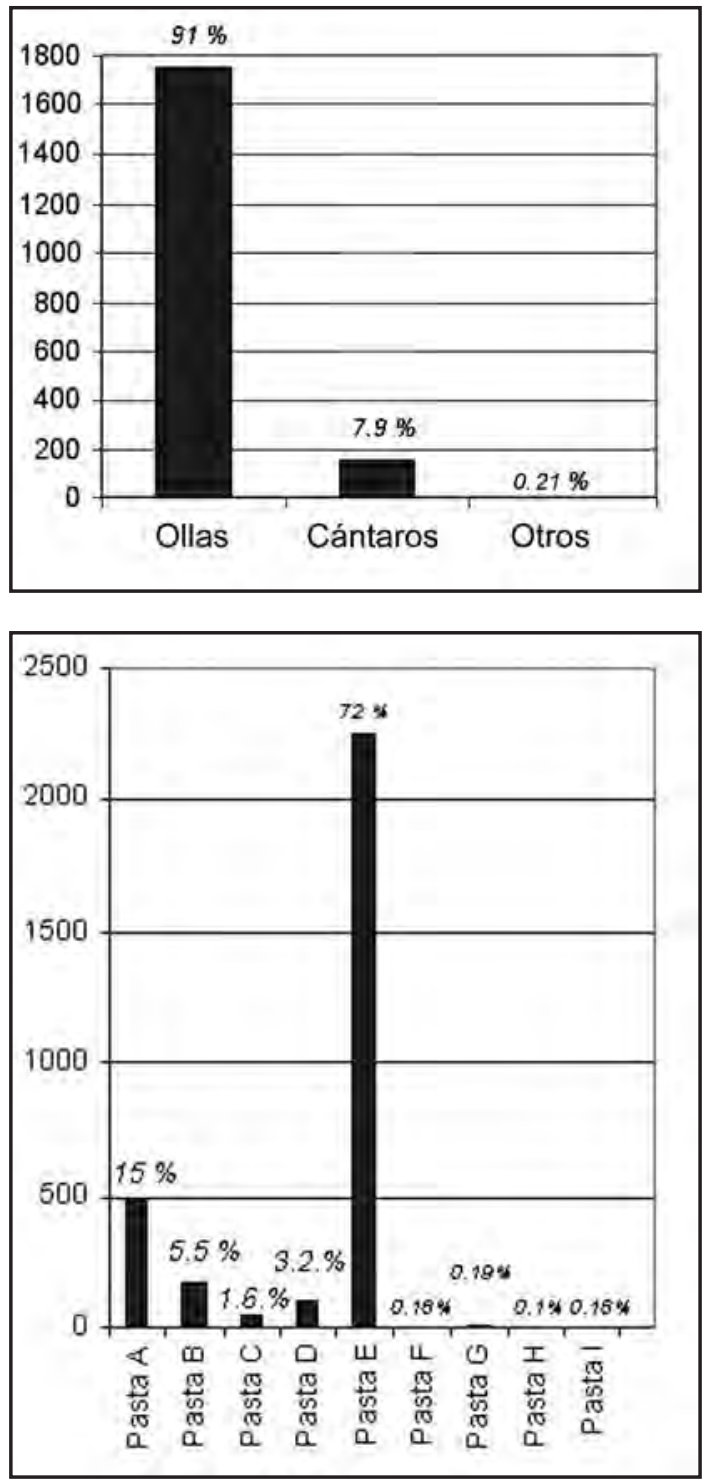

Cuadro 5. Frecuencia de tipos de pasta.

\section{Descripción de Pastas}

El análisis de pasta nos ha permitido identificar 9 tipos de pasta, 4 de las cuales son las más populares (Pasta A, B, D y E) (Cuadro 5). Así mismo hemos podido observar claras correspondencias entre tipos morfológicos y tipos de pastas lo que permitiría definir alfares particulares, lamentablemente no se pudieron realizar los análisis adicionales para completar esta tarea. Los tipos de Pasta A, B, C, D y E corresponden básicamente a las Fases 1 y 2 de Naranjal con las cuales se manufacturaron las vasijas del centro de producción, mientras que las pastas F, G, H e I, se introducen en el asentamiento en la Fase 3 (Fig. 10).

\section{ESCALA DE PRODUCCIÓN}

Hemos identificado un centro de producción, pero definirlo implica seguir criterios como la escala de la producción, el grado de especialización, la intensidad de la producción y el modo de producción. Medir cuantitativamente estos criterios de análisis excede el alcance de este trabajo y se ve impedido por la metodología de la excavación y de la muestra. Sin embargo, podemos acercarnos a estimar la escala de la producción a través del gran número de contextos de desecho registrados en Naranjal, seis fueron hallados en las excavaciones del año 2004 y ocho en las recientes excavaciones del año 2006 (Fig.3). Así mismo, la cantidad de fragmentos de cerámica en cada uno de estos contextos puede superar la cifra de 16000 como fue el caso de la Unidad de Excavación 6, en donde se excavó parcialmente uno de estos contextos. El número de contextos dejados por los alfareros al cierre de sus ciclos de producción nos estaría indicando que la producción de cerámica de estilo Ychsma en Huaca Naranjal se realizó a gran escala durante las fases 1 y 2 del sitio, trascendiendo el consumo local propio.

\section{Naranjal durante el Horizonte Tardío}

Durante la Fase 3 los contextos de desecho desaparecen iniciándose la construcción de arquitectura asociada al edificio principal (Fig.4). Los materiales asociados a estos contextos arquitectónicos proceden de rellenos, y presentan características novedosas con respecto a las fa- 
Figura 10. Tipos de pasta hallados en Naranjal.

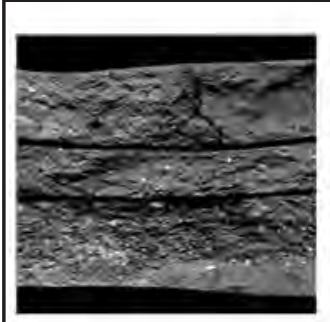

\section{PASTA A}

Tamaño de inclusiones: 0.1 - 0.5

Porcentaje de inclusiones: $5 \%$

Ordenamiento: Muy pobre

Redondez: Sub-redondeado

Esfericidad: Media

Tipo de inclusiones: Cuerpos blancos

cristales y piedras negras

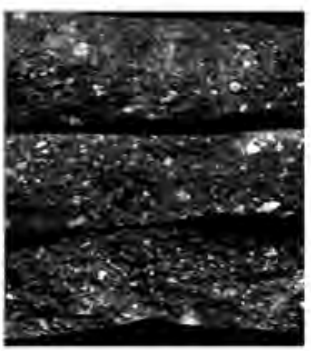

\section{PASTA B}

Tamaño de inclusiones: 0.1 - 1.0

Porcentaje de inclusiones: $10 \%$

Ordenamiento: Pobre

Redondez: Sub-angular

Esfericidad: Media

Tipo de inclusiones: Cuerpos blancos mica y cuerpos negros

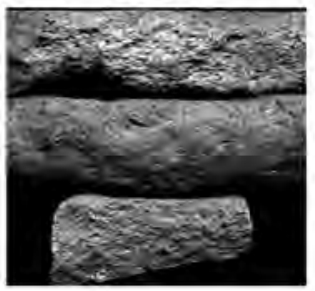

\section{PASTA C}

Tamaño de inclusiones: 0.1 - 0.3 Porcentaje de inclusiones: $3 \%$

Ordenamiento: Muy pobre

Redondez: Sub-redondeado

Esfericidad: Media

Tipo de inclusiones: Arena, cuerpos

blancos, y mica.

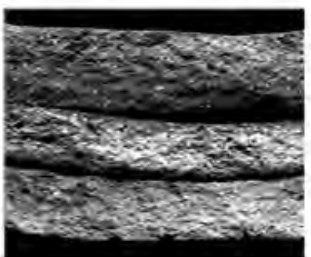

\section{PASTA D}

Tamaf́o de inclusiones: 0.1 - 0.5

Porcentaje de inclusiones: $4 \%$

Ordenamiento: Muy pobre

Redondez: Sub-redondeado

Esfericidad: Media

Tipo de inclusiones: Cuerpos blancos

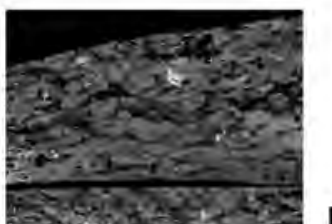

\section{PASTA E 1}

Tamaño de inclusiones: $0.1-2.0$ Porcentaje de inclusiones: $10 \%$

Ordenamiento: Regular

Redondez: Sub-angular

Esfericidad: Media

Tipo de inclusiones: Cuerpos negros,

y cuerpos blancos

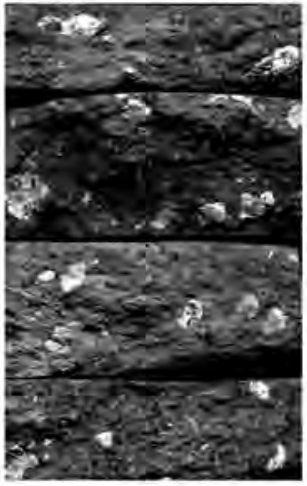

\section{PASTAE 2}

Tamafó de inclusiones: 0.1 - 4.0

Porcentaje de inclusiones: $15 \%$

Ordenamiento: Regular

Redondez: Sub-angular

Esfericidad: Media

Tipo de inclusiones: Grandes cuerpos

blancos, y cuerpos negros

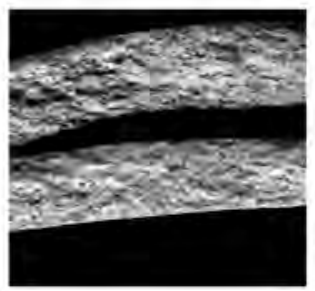

\section{PASTAF}

Tamaño de inclusiones: 0.1

Porcentaje de Inclusiones $0.10 \%$ Ordenamiento: Muy pobre

Tipo de inclusiones: cuerpo marrón ocasional, puntos brillantes

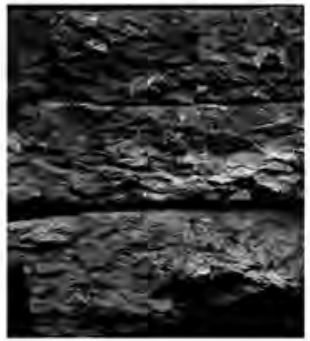

\section{PASTA G}

Tamaño de inclusiones: $0.1-6.0$ Porcentaje de inclusiones: $50 \%$

Ordenamiento: Muy bueno

Redondez: Angular

Esfericidad: Baja

Tipo de inclusiones: Piedras laminares

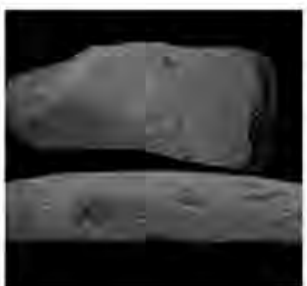

\section{PASTA H}

Tamaño de inclusiones: 0.1

Porcentaje de inclusiones: $0.10 \%$

Ordenamiento: Muy pobre

Tipo de inclusiones: Puntos rojos ocasionales

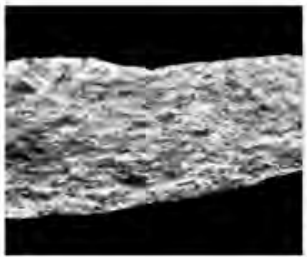

\section{PASTA I}

Tamaño de inclusiones: $0.1-0.5$

Porcentaje de inclusiones: $3 \%$

Ordenamiento: Muy pobre

Redondez: Sub-redondedo

Esfericidad: Media

Tipo de inclusiones: Arena, cuerpos negros 
ses previas. Aparecen fragmentos muy decorados; fragmentos con el diseño de la serpiente; ollas sin cuello; vasijas de servicio, tales como cuencos, uno de los cuales fue hallado completo pero fragmentado sobre el relleno de una banqueta; una cantidad de fragmentos de cocción reductora y de acabado pulido, algunos correspondientes a pequeñas vasijas comúnmente denominadas Chimú-Inca; y varios tipos de vasijas algunas con decoración. Hay que agregar un pequeño aríbalo hallado por Miguel Cornejo en las excavaciones del año 2004.

La arquitectura y los materiales de la Fase 3, indican que el asentamiento sufrió una reorganización interna que implicó al menos un desplazamiento de las áreas de producción para la construcción de arquitectura monumental, durante la Fase Ychsma Tardío B. Hay que considerar que es precisamente durante esta fase que varios asentamientos Ychsma de la Costa Central sufren una reorganización debido a la llegada de los Incas.

\section{Discusión Y CONCLUSIONES}

El presente artículo fue presentado en la mesa Economía y Sociedad del Simposio Ychsma, la cual propuso discutir básicamente cuatro puntos: 1. actividades de producción practicadas por esta sociedad, 2. escala de la producción, 3. control político de la producción, y 4. situación socio-económica de la población que realizaba tales actividades de producción.

Las evidencias indican que Huaca Naranjal fue un centro de producción de cerámica a gran escala, donde se manufacturaba ollas y cántaros del estilo Ychsma de forma especializada y exclusivamente distribuidos para el consumo doméstico. Los contextos de desecho, por su parte, serían resultado de eventos relacionados con el cierre de ciclos de producción como de uso de vasijas domésticas en el asentamiento. La especialización de este centro de producción en la manufactura de ciertos tipos morfológicos de cerámica doméstica indica indirectamente que otros talleres producían otros tipos de cerámica incluyendo los tipos "finos" tales como los cuencos, botellas y cántaros cara gollete. Tal situación revela una división del trabajo entre los talleres de cerámica de la sociedad Ychsma, y una organización socioeconómica basada en la especialización del trabajo al interior de la clase social de los artesanos.

Acerca del control político de la producción, hay talleres en Pachacamac al interior de las PCR (Eeckhout 2004: 406) las cuales indican que grupos de artesanos se encontraban auspiciados por las elites. En Naranjal no tenemos evidencia suficiente para esclarecer este punto, ya que una manifestación de poder político como la arquitectura monumental sólo apareció en la Fase 3 cuando las áreas de producción desaparecen del área o son desplazadas. Sin embargo, el hecho mismo de producir un conjunto restringido de tipos morfológicos para un consumo determinado así como la ausencia total de la producción de cerámica "fina" sugieren que existió cierto control o normatividad en el centro de producción.

Estos resultados nos llevan a una pregunta inmediata sobre la ubicación política de Naranjal, ¿Cuáles son las implicancias de la presencia de un centro de producción de cerámica estilo Ychsma en el valle de Chillón? Se acepta que, durante el Período Intermedio Tardío, los valles de Lurín y Rímac formaban una unidad social denominada Ychsma cuyo centro principal era Pachacámac. Sin embargo iexistieron "enclaves" Ychsma fuera de los límites políticos definidos etonohistóricamente, como parece sugerir el caso de Naranjal? La existencia de un centro de producción de cerámica estilo Ychsma en el valle del Chillón, considerando la carencia de una definición de la cerámica de este valle, nos obliga a reevaluar el carácter de la entidad política denominada Collique, sus espacios políticos y las relaciones con la sociedad Ychsma. 
Si bien hasta la actualidad no existen publicaciones que definan el estilo cerámico asociado a la entidad política Collique (cuyo centro político se encuentra próximo a Naranjal), algunos indicios parecen señalar que la cerámica Collique es morfológica y estilísticamente similar a la Yschma, por lo cual parece que los tres valles de la costa central comparten una misma tradición cultural, aunque bajo dos entidades políticas definidas etnohistóricamente. Si esto es así, no se podría distinguir un componente cerámico Yschma de uno Collique, entonces, ¿a que entidad política corresponde el centro de producción de Huaca Naranjal? El tema de las fronteras ha sido objeto de discusión en este Simposio (ver Tantalean este volumen para el caso sur), si bien la presencia de un estilo cerámico particular en un asentamiento no lo hace políticamente dependiente de la entidad social definida por ese estilo, creemos que un centro de producción de un tipo exclusivo de cerámica a una escala que sobrepasa el consumo local debería llevarnos a reconsiderar las relaciones sociopolíticas que garantizan su existencia y redefinir los espacios de ocupación de la sociedad Ychsma.

\section{BiBLIOGRAFÍA}

Arnold, Dean

1994 Tecnología cerámica andina: una perspectiva etnoarqueológica. En: Tecnología y organización de la producción cerámica prehispánica en los Andes, editado por Izumi Shimada, pp. 477-504. Lima: PUCP.

Bonavia, Duccio

1966 Sitios arqueológicos del Perú (1ra parte). En: Arqueológicas, N 9, Publicación del Instituto de Investigaciones Antropológicas. Museo Nacional de Antropología y Arqueología, Lima.

Carmichael, Patrick

1994 Cerámica Nazca: producción y con- texto social. En: Tecnología y organización de la producción cerámica prehispánica en los Andes, editado por Izumi Shimada, pp. 229-247. Lima: PUCP.

Cerdan y Pontero, Ambrosio

1793 "Tratado sobre las aguas de los valles de Lima”. En: Mercurio Peruano. Tomo VII, Lima.

Cornejo, Miguel

2004 Informe Final del Proyecto de Evaluación Arqueológica Huaca Naranjal. Presentado al Instituto Nacional de Cultura.

2006 Informe Final del Proyecto de Evaluación Arqueológica Mercado Naranjal. Presentado al Instituto Nacional de Cultura.

Eeckhout, Peter

2004 La sombra de Ychsma: ensayo introductorio sobre la arqueología de la costa central del Perú en los periodos tardíos. Bulletin de l'Institut français d'études andines, 33(3): 403-423

FAUA-UNI-FORD

1989 Inventario del Patrimonio Monumental Inmueble, Lima, valles de Chillón, Rímac y Lurín. Facultad de Arquitectura, Urbanismo y Artes UNI. Lima: Fundación Ford, Proyecto 01.

Horkheimer, Hans

1965 Identificación y Bibliografía de importantes sitios prehispánicos del Perú En: Arqueológicas, Nº 8, Publicación del Instituto de Investigaciones Antropológicas. Museo Nacional de Antropología y Arqueología, Pueblo Libre, Lima.

Ludeña, Hugo

1975 Secuencia cronológica y cultural del valle del Chillón. Tesis para optar el grado de Doctor. Programa de Arqueología de la Universidad Nacional Mayor de San Marcos. Lima. 
Milla Villena, Carlos

1974 Inventario y Catastro del valle del Rímac y Santa Eulalia. Instituto Nacional de Cultura, Centro de Investigación y Restauración de Bienes Monumentales, Lima.

Ravines, Rogger

1985 Inventario de Monumentos Arqueológicos del Perú. Lima Metropolitana (Primera aproximación). Instituto $\mathrm{Na}$ cional de Cultura. Municipalidad de Lima Metropolitana, Lima.

Rice, Proudence

1987 Pottery Analysis: A Sourcebook. Chicago: The University of Chicago Press.

Shimada, Izumi

1994 Tecnología y organización de la producción de cerámica prehispánica en los Andes. Lima: PUCP
Tosi, Joseph

1960 Zonas de Vida Natural en el Perú. Memoria Explicativa sobre el Mapa Ecológico del Perú. Lima. IICA-OEA.

Turner, W y José Bravo

1907 Informes sobre el Río Chillón. Lima. Imprenta de "El Lucero".

Vallejo, Francisco

2004 El estilo Ychsma: características generales, secuencia y distribución geográfica. Bulletin de l'Institut français d'études andines, 33(3):595-642 